\title{
A Virtual Touch Interaction Device for Immersive Applications
}

\author{
Paulo Gallotti Rodrigues, Alberto Barbosa Raposo and Luciano Pereira Soares
}

Tecgraf (Computer Graphics Group) / Dept. of Informatics / PUC-Rio (Pontifical Catholic University of Rio de Janeiro)

\begin{abstract}
Traditional interaction devices such as computer mice and keyboards do not adapt very well to immersive environments, since they were not necessarily designed for users who may be standing or in movement. Moreover, in the current interaction model for immersive environments, based on wands and 3D mice, a change of context is necessary in order to execute non-immersive tasks. These constant context changes from immersive to $2 \mathrm{D}$ desktops introduce a rupture in user interaction with the application. The objective of this work is to study how to adapt interaction techniques from touch surface based systems to 3D virtual environments to reduce this physical rupture from the fully immersive mode to the desktop paradigm. In order to do this, a wireless glove (v-Glove) that maps to a touch interface in a virtual reality immersive environment was developed, enabling it to interact in 3D applications. The glove has two main functionalities: tracking the position of the user's index finger and vibrating the fingertip when it reaches an area mapped in the interaction space to simulate a touch feeling. Quantitative and qualitative analysis were performed with users to evaluate the v-Glove, comparing it with a gyroscopic 3D mouse.
\end{abstract}

Index Terms - 3D user interaction; immersive environments; optical-tracking; wireless glove; tactile feedback;

\section{INTRODUCTION}

In recent years, several research initiatives in the field of virtual reality (VR) have focused on the development of interaction techniques for the selection and manipulation of objects, navigation and wayfinding [1][2][3]. Techniques for controlling application parameters were also studied, allowing the change of scalar values and the choice among alternative menu options. However, interfaces for system control in virtual environments have not yet been studied in depth and are still an open area of study [4].

Since interface controls are an important part of conventional desktop interfaces, adapting these controls for virtual environments is necessary. A limited number of control widgets, like buttons, can be easily accessed, but that alone does not adequately suffice for more complex situations such as selection, menu navigation, and alphanumeric input. Thus, all solutions that enable 2D menus adapted to immersive environments face the problem of effectively reaching a menu item in the interaction space [5].

Manuscript Received on July 10, 2011

E-mail: lpsoares@gmail.com
Some devices were created specifically for immersive environments, such as wands, gloves and 3D mice, providing a more appropriate interface to the user in this kind of environment. However, these devices have a high cost, require an often-complex infrastructure (cables, cameras and sensors) and have limitations when faced with situations common in the WIMP (Windows, Icons, Menus and Pointers) paradigm.

Thus, the user is forced to make several changes of context every time it is necessary to accomplish a task which is not supported in immersive mode. These constant changes in the context of immersion signify a break in the way a user interacts with the application. Furthermore, these changes often force the users to disassemble the immersion apparatus like the HMD (Head Mounted Display) and gloves, forcing them to sit at a table to perform the interaction tasks necessary before then returning to the immersive environment. This restricts the use of immersive applications over longer periods of time or demands support from a second person to execute WIMP related tasks.

When considering the current advances achieved by interfaces based on single or multi-touch gestures, it was realized that there is a potential to use such concepts in immersive virtual environments. The literature presents many initiatives towards introducing mobile devices as interaction devices in virtual environments [6][7]. This is mainly due to the fact that modern mobile devices generally have these modern interface resources integrated, and smartphones and tablets are considered quite simple and useful for gesture interactions. However, they still require the user to hold the device when interacting with the immersive environment.

Based on these ideas, the aim of this paper is to explore the possibilities of using a touch-like interface in an immersive virtual reality environment using a virtual touch screen mapped in the 3D space. The advantages and disadvantages of this approach were investigated by comparing this strategy to standard interaction techniques in this class of environments. A glove called v-Glove was built using electronic components and a 3 DOF (degrees of freedom) IR (infrared) optical tracking system. The cameras used were those integrated in the Wii Remote control, also known as WiiMote. Actuators were used to create the vibration sensation and radio transmitters allowed for communication between the glove and the computer. Based on these features, the v-Glove is intended to simulate the feeling of touching within a virtual immersive environment.

The operating principle of the v-Glove is based on tracking 
the position of the index finger and mapping it to $\mathrm{X}$ and $\mathrm{Y}$ display coordinates, similar to a traditional desktop mouse. By moving the finger to the right, for example, the mouse pointer moves on the screen following this same direction. The operation of clicking and dragging objects is based on the $\mathrm{Z}$ reference axis. When bringing the fingertip to a predetermined point in $\mathrm{Z}$, the system detects the intention to click on the object selected at that moment. To reproduce the movement of dragging, the user has to keep the fingertip in the touch area while moving his hand in the $\mathrm{X}$ and $\mathrm{Y}$ axes.

A proof of concept was conducted with the tasks of navigation in CAD models, selection of objects and interaction with menus adapted for the software. Qualitative and quantitative analyses were conducted with users divided into groups according to a profile of experience in the use of 3D applications. All tests and interviews were recorded for further more detailed analysis.

The remainder of this paper is organized as follows. Section 2 describes the relevant works that serve as reference and inspiration for this research. The proposed solution is presented in details in section 3, its software architecture in section 4, and interface adjustments in section 5. Section 6 presents a case study of the proposed solution with the results presented in section 7. Section 8 presents the conclusions and future work proposals.

\section{RELATED WORK}

This research was inspired by several projects from different research areas. For this reason, the related work was divided in three subsections according to the area considered to be related to the most significant contribution of each related work.

\subsection{Tactile feedback}

The system proposed by Scheibe, Moehringer and Froehlich [8] is a tactile feedback device manipulated by the user's fingertips for an immersive virtual reality application. The system consists of wired thimbles involving the tips of each finger. In order to simulate the contact with a surface, the wire length is shortened to make them to come into direct contact with the fingertips and a vibration is caused in the wire to enhance the user's perception of a tactile stimulus. Both the shortening and relaxation of the wires as the process of vibration is controlled by a micro-controller that receives commands from a virtual reality application. Studies have shown that users preferred the tactile feedback version of the system over other versions without feedback, especially when performing direct manipulation tasks. Bullion and Gurocak also presented a compact force feedback glove in [9]. Their main objective was to reduce the size and the number of actuators in a haptic glove, increasing user ability to interact with virtual environments.

Our proposal has the advantage of an absence of wires between the glove and the tracking system, which improves user mobility, especially considering the context of highly immersive environments.

Tactile displays can improve interaction in devices as pre- sented in [10]. A series of experiments were conducted based on the development of perceivable tactile icons (called tactons). These tactons are designed to have parameters like: amplitude modulation, frequency, and waveform, each of which is capable of creating distinctive effects. This resource improved interactions with mobile application displays. The goal of this research was conveying two-state signals such as 'on/off', 'slower/faster', or 'left/right'. The results showed a reduction of the visual burden experienced when users interact with mobile applications.

\subsection{Finger Tracking Systems}

A.R.T. makes tracking systems for immersive virtual reality environments and has a specific device for finger tracking [11]. This glove allows the tracking of user hand orientation and of the position of up to five fingers at the same time. The communication between the glove and the control center is wireless. A pair of infrared (IR) LEDs is used on each finger for tracking. Each of these sets of LEDs emits light at a specific timing, thus allowing the cameras to identify the fingers individually. This solution has no tactile feedback integrated. Grossman, Wigdor and Balakrishnan [12] proposed a similar approach for finger tracking based on IR markers used for interaction with objects in a $3 \mathrm{D}$ volumetric display.

\subsection{Virtual Touch Screen}

Tosas and Li [13] proposed a mixed reality environment that allows users to interact with a virtual touch screen. Techniques of computer vision are used to detect the hand and gestures of typing on a virtual keypad. The idea is that users can interact with elements on a graphical user interface by using bare hands to click on windows, icons and menus similar to windows managers traditionally used in desktop environments. The proposed system consists of an HMD that allows the user to visualize the virtual keyboard, a camera to capture the hand movements and the software to identify and track hand position, which also generates an audible and visual response when keys are pressed. The click detection is done by identifying the shortening of a finger in the image, when the finger was bent.

Lindeman, Sibert and Hahn [14] proposed 2D interaction based on hand-held windows in virtual immersive environments. They created a testbed called HARP (Haptic Augmented Reality Paddle) whose main idea is to take advantage of the bimanual interaction in a passive-haptic feedback using a physical surface similar to a track pad. The user holds the paddle in his non-dominant hand and touches the surface to interact with the system. 2D UI widgets are drawn on the face of the virtual paddle representing its current physical location. A head tracking system is also part of the proposed solution to adjust the view relative to the user field of vision. The results of the study support the utility of hand-held windows as a general interaction tool, assuming they move with the user and can be positioned to allow the user to work effectively. With the added support provided by passive-haptic feedback, hand-held windows can provide an effective interface for manipulating 2D widgets. When comparing our work to these ideas, our work has the advantage of not requiring the user to carry the physical 
paddle in one hand, as the virtual touch surface can be activated or deactivated when necessary.

Current research on interaction techniques for immersive environments is focused on selection, manipulation, navigation and wayfinding. The literature includes several papers regarding system configuration, symbolic input and 3D menu navigation. This paper presents an interaction device designed to perform application control tasks inspired by techniques already used with success in other research areas, such as the idea of touch surfaces and haptic feedback. The proposed device was developed as a glove with tactile feedback for interaction with a virtual touch screen mapped in a VR immersive environment.

\section{SOLUTION}

The purpose of this research is to study the advantages of applying similar concepts of touch and multi-touch interfaces on immersive virtual reality applications. The main focus was the development of a glove to interact with a virtual touch screen in an immersive projection environment. The hardware view of the solution developed is shown in Fig. 1. All built elements were designed to be used in environments without tables for keyboards or mice, while retaining other ergonomic priorities for immersive environments, like the need for user mobility and low luminosity.

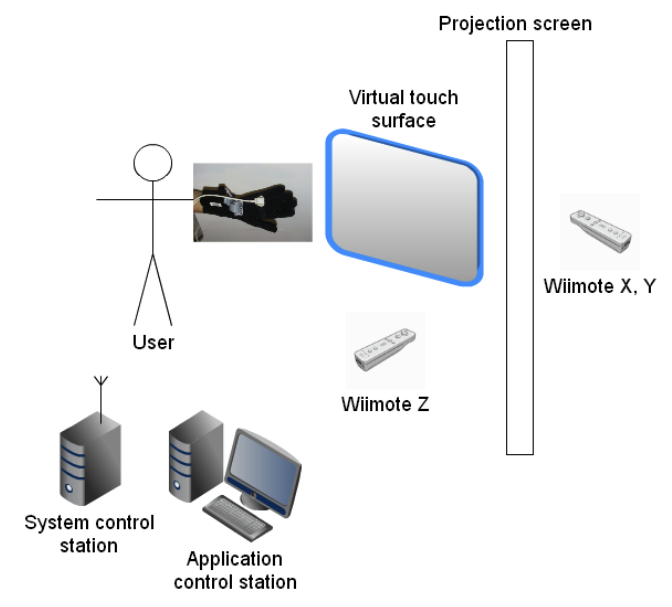

Fig. 1. Schema of the v-Glove hardware.

\section{$3.1 v$-Glove (Tactile Feedback)}

Different approaches can be considered when designing a glove for interaction, as presented in the survey of glove-based systems in [15]. The v-Glove is made of micro fiber and has been tailored to ensure a proper size for a large number of users as presented in Fig. 3(a). The choice of fabric took into account its thickness and flexibility as it is crucial that the glove does not restrict the movement of the users' hands or their ability to handle a tracked wand. It is important that the glove does not warm the user's hand, even if the device is used in temperature-controlled environments. Additionally, the black color helps the contrast of the optical tracking system.

\subsection{1) Arduino Micro-controllers}

The Lilypad Arduino micro-controller is a version of the well-established processor Arduino developed by SparkFun Electronics for use in clothing [16]. The programmable microprocessor model Arduino Lilypad Main Board 328 is presented in Fig. 2(a). The XBee modem is a component supplied by Digi International Inc. and can be coupled to a Lilypad XBee shield for use in wearable circuitry [17]. XBee implements the ZigBee protocol for wireless communication and has a $1 \mathrm{~mW}$ microchip antenna with a range up to 100 meters and data transmission rate of $250 \mathrm{kbps}$ (Fig. 2(b)). The Lilypad Vibe Board component is an actuator capable of vibrating when powered by 5 volts. It is also built into a wearable configuration as shown in Fig. 2(c).

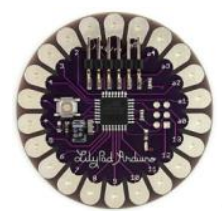

(a)

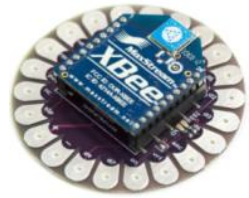

(b)

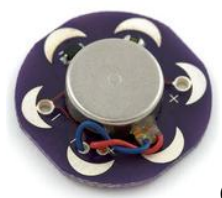

(c)
Fig. 2. (a) Lilypad Arduino, (b) XBee and (c) Vibe Board [15].

The XBee module mounted in the v-Glove is configured as a receiver. Another identical module was connected to the control station via USB port, configured as a transmitter. Upon detecting a touch event, the application executed by the control station sends a command to the transmitter XBee, which then forwards it to the receiver XBee attached to the v-Glove.

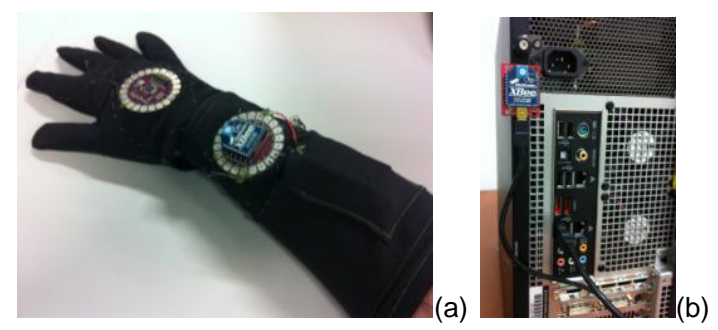

Fig. 3. (a) v-Glove Prototype, (b) Modem in the Control Station.

The electronic components responsible for the tactile feedback sub-system were tailored on the glove. The stitching of the components was done using a sewing line with conductive properties specially created for this purpose. The seam line is silver-plated and has electrical resistance of approximately 45 Ohms per meter. Fig. 4 shows the schematic circuit stitched on the v-Glove.

A $9 \mathrm{~V}$ battery provides power for all components of the circuit. The XBee modem receives, through its antenna, the commands from the XBee modem transmitter. The command is then sent to the transmit port ( $\mathrm{Tx}$ ) which is connected directly to the receive port $(\mathrm{Rx})$ of the Lilypad Arduino microcontroller. The software stored on the micro-controller receives this information and sends signals to pin number 7, which is directly connected to the vibe board. Activating this device causes a feeling of a slight vibration in the user's fingertip. The duration 


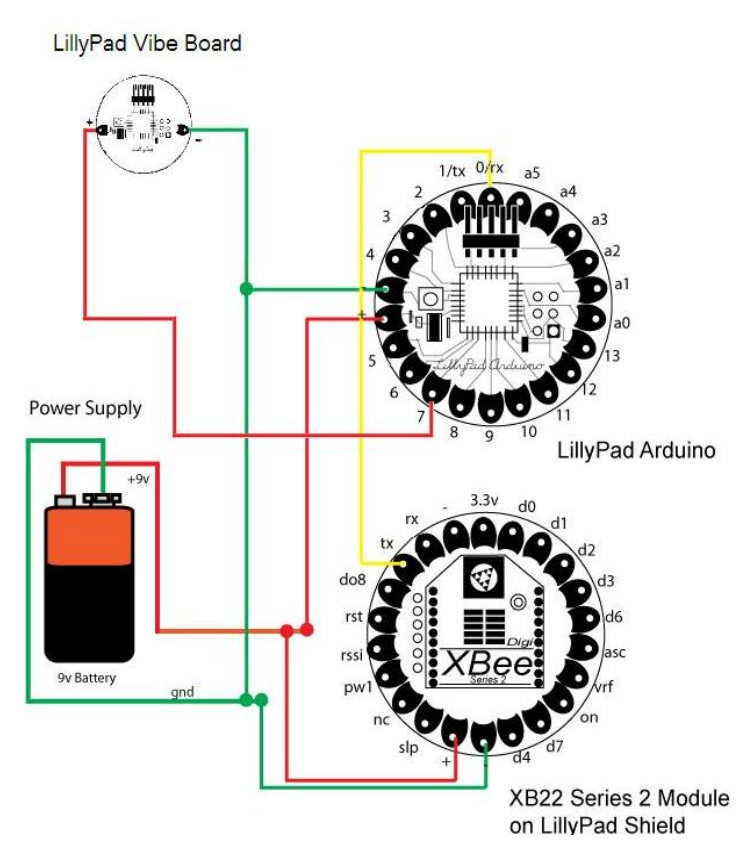

Fig. 4. v-Glove Circuit Diagram [18].

of vibration may be controlled.

The main principle of the haptic feedback subsystem is to provide a vibration when the user reaches the touch area mapped by the cameras in the physical space. The vibration is active for 300 milliseconds and is interrupted even if the user keeps the finger in the so called click area. Tests that maintained the vibration while user's finger is in the click area were conducted, but an empirical evaluation considered the vibration intensity uncomfortable for the user when active for longer periods of time. Regarding cursor operation, positioning the fingertip in the click area causes a cursor (mouse) pressed event to be generated in the application. The click operation is only completed when the user returns his finger to a position outside the click area, generating a cursor-released event in the operational system. This approach is necessary to enable drag-based interactions, when there should be a way to inform the system when a movement starts and when it ends.

\subsection{2) Application running in the microcontroller}

The program that runs in the Arduino is called "sketch", and it is written in a programming language similar to $\mathrm{C}$. The structure of a sketch can be divided into three parts: an area for declaration of variables and functions created by the user; the setup() function; and the loop() function.

The setup function is the first to be called when the program starts. It can be used for the initialization of variables and controller ports or for loading third-party libraries. The setup function is executed only once, at startup or when the board is reset. The loop function is called continuously during program execution. The first execution of this function occurs only after the end of the setup function. Below is a simplified sketch with the source code used in the v-Glove:
1 int finger1Pin $=7$;

2 int incomingByte $=-1$;

3

4 void $\operatorname{setup}()$

5\{

6 Serial.begin(9600);

7 pinMode(finger1Pin, OUTPUT);

8 Serial.flush();

9 \}

10

11void loop()

12\{

13 if (Serial.available ()$>0)$

14 \{

15 incomingByte = Serial.read () ;

16

17 if (incomingByte == ' 1 ')

18\{

19 digitalWrite(finger1Pin, HIGH);

20 delay(300);

21 digitalWrite(finger1Pin, LOW);

$22\}$

$23\}$

$24\}$

Line 1 makes the declaration of variables related to the port of the Arduino microcontroller used (in this case port number 7). Line 2 declares the variable used for reading bytes from the serial port. Lines 6-8 define the mode of operation for the ports used as output.

Line 13 runs the code block if there is information to be read into the serial port, which is read in line 15 . Line 17 performs the following blocks of code if the byte read is 1 . Finally lines $19-21$ write a signal of high voltage $(5 \mathrm{~V})$ in the output port, wait 300 milliseconds, then remove the signal from that port.

\subsection{3) Finger identification}

In order to track the finger position, an infrared optical tracking solution is used. Out of the several options evaluated, the 8910 3M reflective tape was selected as an infrared marker. This tape is widely used as an element of urban signs due to its high reflective properties for visible light and its high flexibility (for example it can be easily tailored to the uniform of traffic agents).

The $3 \mathrm{M}$ retro-reflective tape was then applied to the tip of the index finger of the glove. This tape is able to reflect light with greater intensity in the direction of the illuminating source. With this tape, it is possible to efficiently reflect IR to the camera if an illuminator is positioned close to the cameras. The IR cameras capture the light reflected by the tape and estimate the position of the user's finger. As the proposed system uses two perpendicular IR cameras, it is important that the retro-reflective tape is visible by both cameras. The most appropriate way to position the tape in order to ensure good visibility is to attach them to the fingertip. The positioning of the cameras in relation to the user and the tape applied on the glove are shown in Fig.5. 


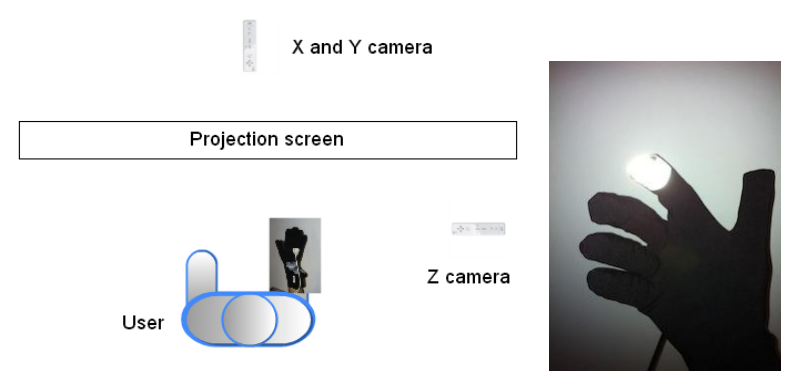

Fig. 5. Set-up of cameras and tape applied on the glove.

\subsection{Tracking}

The camera chosen for the tracking system is the one integrated to the remote control of the Wii video game. This camera has a built in filter to capture only the infrared range, with greater sensitivity to wavelengths near $940 \mathrm{~nm}$. The WiiMote uses bluetooth technology for wireless communication. The experiments developed by Chung Lee [19] showed that the resolution and precision of the device is enough for tracking even small points. The specification was unpublished, but it appears that the camera has resolution of 1,024×768@100Hz, and a 45-degree horizontal field of view. The device can track up to four points simultaneously by the integrated hardware, minimizing data transmission over the Bluetooth connection and simplifying development of algorithms.

The main advantages of using the WiiMote instead of a conventional camera are that it: is wireless, already has a built in IR filter, and has development libraries that provide the $\mathrm{X}$ and $\mathrm{Y}$ coordinates of the IR points captured. A disadvantage of the WiiMote is that it is able to identify only four points of infrared light simultaneously, which may limit an application that is intended to track all the fingers. This will not be a problem in our case, since the proof of concept proposed in this research only uses the index finger.

As the camera of the WiiMote provides the coordinates of the points in a two-dimensional plane, a second camera was incorporated and positioned laterally to capture the depth axis. Thus two perpendicular cameras compose the final solution.

The first camera is responsible for tracking the axes $\mathrm{X}$ and $\mathrm{Y}$. It indicates the position of the user's fingers on these two dimensions. The second camera is responsible for the $\mathrm{Z}$ axis and it is positioned at the side of the user to map the virtual touch surface. When the user's index finger approaches the mapped plan, the software recognizes a touch on the surface and triggers an event of tactile feedback to the v-Glove, vibrating the user's finger.

Other tracking algorithms using epipolar geometry were evaluated [20][21]. They are very useful for tracking complex objects with multiple markers on it, but require several calibration steps. For this research, only a single point is necessary, therefore the orthogonal WiiMote cameras' placement provides a more practical solution. One advantage of the perpendicular cameras is that, even if the alignment of the cameras is not perfect, the system will continue to work.
The WiiMote infrared camera is only sensitive to a certain intensity and wavelength of infrared light. Considering that this application will be used in an immersive environment with low light, an infrared illuminator component is essential. The created prototype consists of eight infrared LEDs of $5 \mathrm{~mm}$ and a dominant wavelength of $940 \mathrm{~nm}$ fed by a power supply of 12 volts. The LEDs used are of the same type used for remote control devices, and can be easily found in electronics stores. This component generates infrared light that is reflected by the retro-reflective tape on the v-Glove and then captured by the WiiMote cameras.

An alternative to using an infrared illuminator is to place the LEDs directly at the tip of the glove's fingers, instead of the retro-reflective tape. In this case, the camera of the WiiMote would directly capture the light emitted by the LED. The problem with this approach is that the presence of LEDs on the glove increases battery consumption, thus reducing its autonomy.

The LEDs used were evaluated to ensure that the infrared emission was not harmful to users; the values calculated are far below those considered hazardous.

\subsection{Control Station}

The control station is a regular PC running the developed software to track the v-Glove and activate the tactile feedback. This system is responsible for both calculating the coordinates $\mathrm{X}, \mathrm{Y}$ and $\mathrm{Z}$, and also for activating the tactile feedback subsystem. In addition, the software can interact with the final applications in the immersive environment.

\section{SOFTWARE ARCHITECTURE}

The software architecture is divided into five main modules that interact to provide all the features necessary for the v-Glove operation. The application to validate the proposal was developed in Java. Third party libraries were used and they are mostly open source or have consent for use in non-profit research projects.

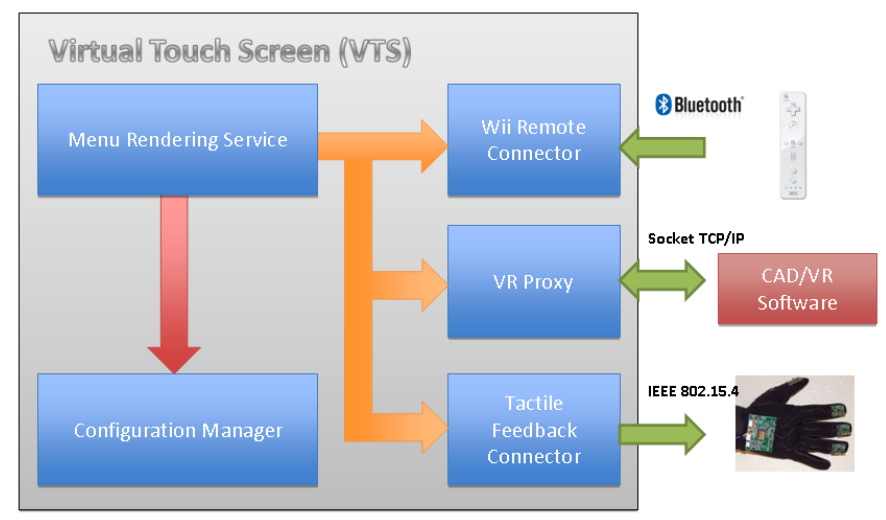

Fig. 6. Software Architecture Diagram. (Color Plate 1)

The architecture diagram of Fig. 6 shows the main modules and the external components with which they interact, indi- 
cating in each case the protocol used. The VR proxy component interacts with the VR/CAD application through a TCP/IP connection. The WiiMote connector performs its communication via the Bluetooth protocol, while the Tactile Feedback connector communicates with the glove through the ZigBee protocol (IEEE 802.15.4).

\section{MENU AND WIDGET INTERFACES}

In order to evaluate the usability of the v-Glove, a case study was prepared for at a CAD application [22]. A list of graphical user interface components was classified and prioritized according to their use in the CAD application to incorporate the following elements: button, checkbox, slider, combo box and text box. These components were then adapted for their use with the v-Glove. Fig. 10 shows the resulting components in the application menus.

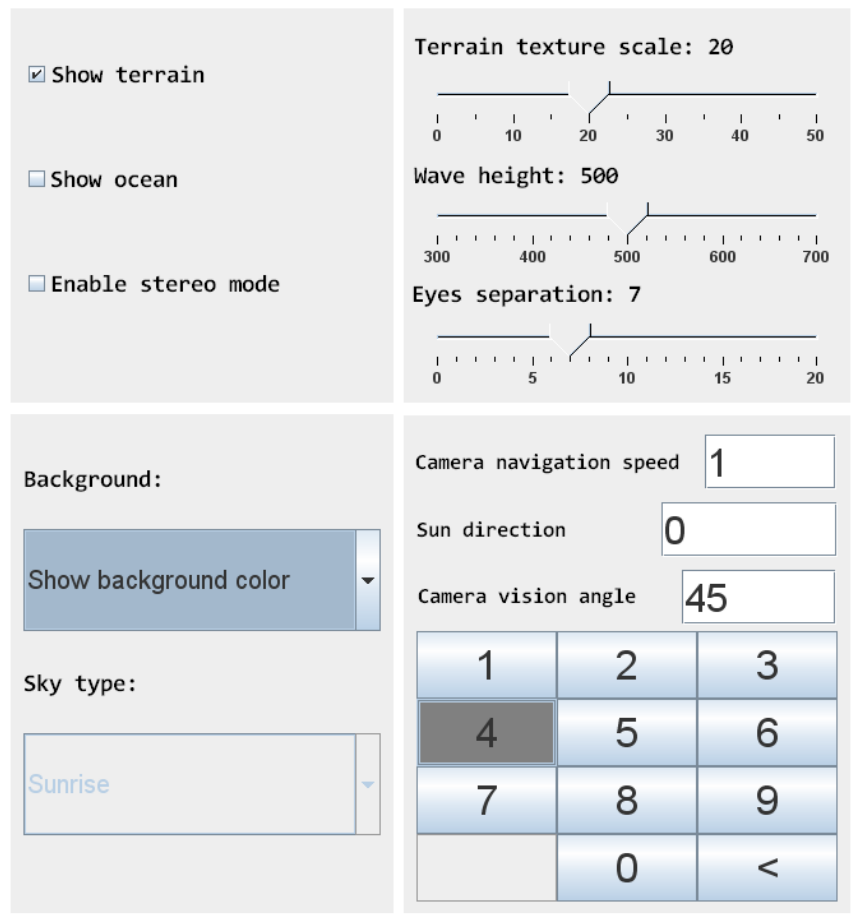

Fig. 7. Widgets Developed for Evaluation Tests.

As a way to complement the tactile feedback, a change in the behavior of the mouse pointer was performed. Depending on the distance of the v-Glove from the touch interface, the pointer on the screen changes its colors. Fig. 8 shows possible colors of the pointer.

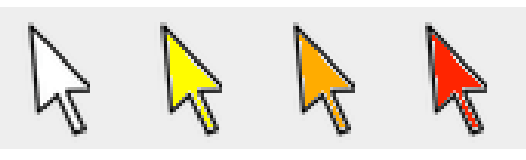

Fig. 8. Pointer colors.

\section{EVALUATION}

The v-Glove was evaluated using the CAD model presented in Fig. 9. This evaluation included three groups of participants with different profiles regarding their development and use of 3D applications.

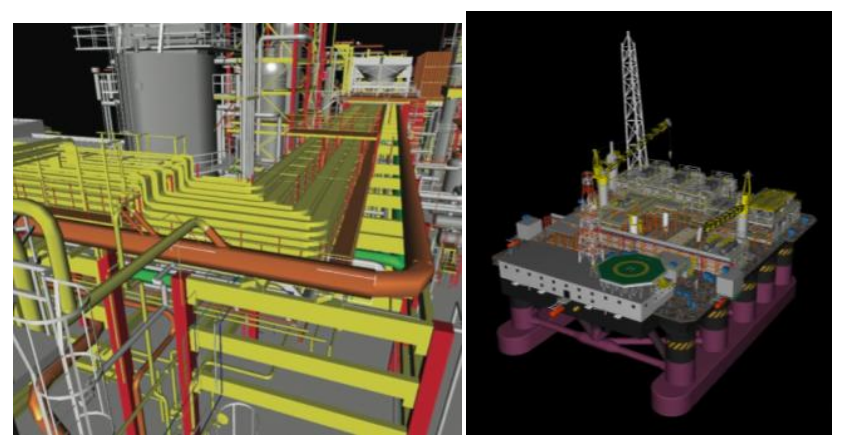

Fig. 9. Virtual Reality / CAD Application. (Color Plate 2)

CAD applications are usually designed for use on the desktop. They are generally not yet fully adapted for immersive environments because their menus and controls are based on the standard WIMP style. All interaction with the software is done through 2D menus and controls (Fig. 10), which required an adaptation of context for its use in the interaction paradigm presented here.

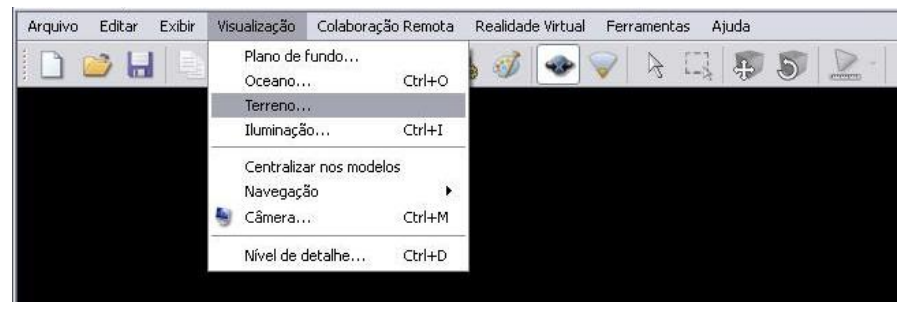

Fig. 10. CAD Application Menu Interface (in Portuguese).

The CAD application menus and widgets were then modified to a more suitable format for interaction with the v-Glove in an immersive environment (Fig. 7). This adaptation occurred only in the relevant components for the evaluation performed during this research.

\subsection{Participants Profile}

Table 1 shows the average age of participants per interest group. Ages varied from 21 to 60 years and, from the eighteen participants, sixteen were male (89\%) and two female $(11 \%)$. All participants were right handed and had the habit of using the mouse with their right hand. This is an important point to be observed, since the v-Glove prototype was designed for right hand only.

Users were divided into three groups of equal size, according to their prior experience in 3D applications: Group E was composed of system analysts who had worked for at least 6 months in the development of the CAD/VR application used; 
Group O was composed of systems analysts with some experience in software development for computer graphics; Group $\mathrm{N}$ was composed of individuals without experience in developing or using graphical software.

Based of the metrics developed by Nielsen [23], Virzi [24], and Lewis [25] it is known that $80 \%$ of usability findings are discovered after five participants and including a larger number of participants does not significantly improve the results. That is why the analysis centered on 3 groups of 6 people each.

TAB. 1. USERS AGE.

\begin{tabular}{|c|c|c|c|}
\hline & Age Average & Youngest & Oldest \\
\hline Global & 29 & 21 & 60 \\
\hline Group E & 25 & 22 & 28 \\
\hline Group O & 31 & 26 & 37 \\
\hline Group N & 31 & 21 & 60 \\
\hline
\end{tabular}

Out of all the participants, 94\% had indicated having some experience with 3D applications,i.e. games, 3D modeling tools, or CAD viewers. All users in Group $\mathrm{O}$ mentioned some knowledge of the CAD/VR tool, but only through superficial contact and not qualifying as part of the CAD/VR developer/user group.

\subsection{Resources}

The simulation room has two ProjectionDesign evo22sx+ projectors configured in passive stereo through a circular polarizing filter with a 120" anti-depolarizer screen. The computer used has a NVIDIA Quadro FX 4600, a 3.2 GHz Intel Core i7 processor with 12 GB of RAM. Fig. 11 shows the room.

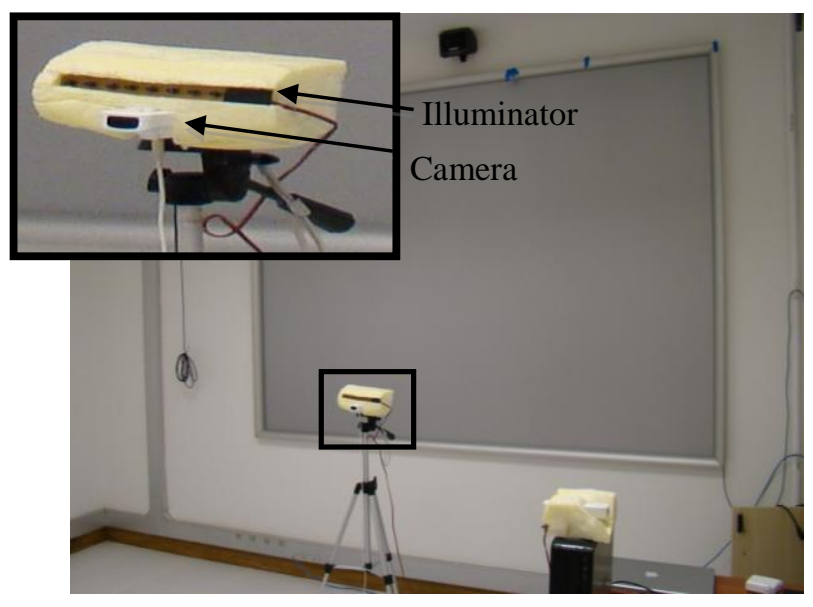

Fig. 11. Evaluation Facility.

\subsection{Evaluation Procedures}

A pilot test was conducted with users with the characteristics of "Group N", as this group was expected to experience more difficulties in the testing process, therefore helping to improve the evaluation process. The main experiment was evaluated by two questionnaires, one issued before the simulation and the other issued immediately after. The examiner completes an accompanying sheet during the operation. All participants completed and signed a consent form that explained the pur- pose of the tests, their role in the process and provided the option of quitting the experiment at any time if they wished.

The evaluation was divided into three sequences of five tasks to be performed by participants using different devices in each sequence. One sequence is performed with the v-Glove with tactile feedback feature turned on, the second sequence is performed without the use of tactile feedback and the other sequence performed with a gyroscopic 3D mouse [26]. Users had a two-minute training phase before using each device.

The five tasks for each sequence are based on the menus in Fig. 7. In the first task, the user was asked to navigate in a CAD scene and look for a particular object, having to select it. In the second task, the user needed to select one of the checkbox options and confirm. The third task involved the selection of a combo box. The fourth task was to select the required value in a slider-type control, and in the fifth and final task, the user was asked to correct the value typed into a textbox, entering a new numeric value and pressing the finish button.

After concluding the sequence of tasks, the interaction device is changed and the sequence is repeated. This was done in a random order to avoid annoying the user by requiring them to perform exactly the same sequence of tasks every time. In order to reduce the learning effects between tasks, the order of use of the devices did not repeat among users of a same group. Considering that there were three devices and six users in each group, it was possible to determine a different order for each user within the group.

\section{RESULTS}

Tullis and Albert [27] present ten usability study scenarios and the related usability metrics that apply to each type of study. According to their classification, the present research fits into the "comparison of alternative designs" and five metrics are applicable: task success, task time, issue-based metrics, self-reported metrics and combined and comparative metrics.

\subsection{Task Success}

The first analysis captured data on the success or failure in performing the tasks required. Most users have no difficulty in completing the tasks. $100 \%$ of users completed the tasks performed with the 3D mouse while using the glove with tactile feedback the success rate of task 2 was $89 \%$, and when the glove without tactile feedback was used the success for tasks 2 and 3 were $94 \%$.

\subsection{Task Time}

The average time for each group is presented in Fig. 12. The glove without tactile feedback has the worst average performance, followed by the glove with tactile feedback and finally the 3D mouse. Moreover, the time to accomplish tasks decreases as the level of experience in 3D of the users. 


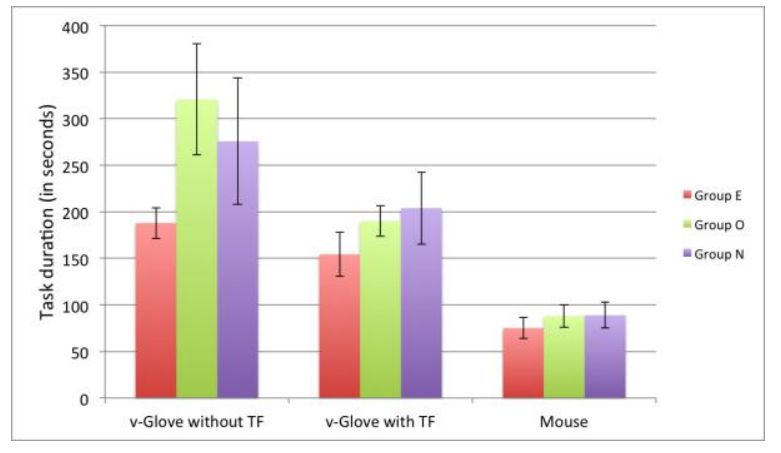

Fig. 12. Task Time Average per Group.

\subsection{Issue-based Metrics}

During the experiment, the evaluators collected information on situations that occurred during the execution of tasks. The issues were classified by frequency, priority (high, medium or low), category, and rating (frequency + priority). Fig. 13 shows the four usability issues ranked with the highest rating among all observed during the experiment.

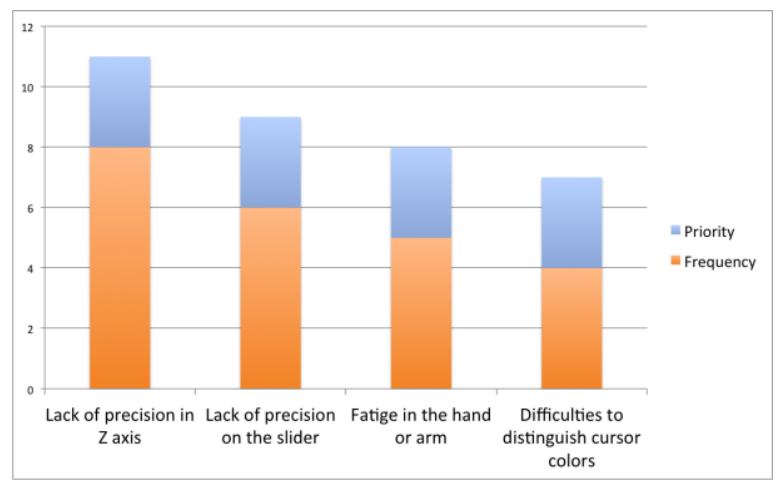

Fig. 13. Usability issues per frequency and priority.

The main problems were:

- $\quad$ Lack of precision in $\mathrm{Z}$ axis - when moving the hand to reach the virtual touch surface it is difficult for the user to prevent variations in the $\mathrm{X}$ and $\mathrm{Y}$ axis. For this reason, when the pointer reaches the click area its position might be different from the original one, causing the click to be performed in a location different than expected;

- Lack of precision on the slider - users reported difficulties with placing the slider indicator in the correct position. This happens because, depending on the used scale, the distance between two markers on the rule was small enough so minimal variations in finger position prevent its placement in the desired location;

- Fatigue in the arm and hand - the suggested position to use the v-Glove requires the user to keep his or her arm raised, causing some users to complain about fatigue in the arm and hand;

- Difficulties in distinguishing cursor color - users reported difficulties in noticing the different pointer colors, particularly between orange and red. This resulted in perception issues in the visual feedback, especially when using the v-Glove without tactile feedback.
The results of the usability issues metric indicate that $58 \%$ of issues observed during the tests are related to problems in the operation of the interaction devices. Regarding the percentage of the usability issues, only $7 \%$ of them are unique to the mouse, while $72 \%$ happen in either versions of the glove. The remaining $21 \%$ refer to issues observed in both devices, like posture problems or fatigue situations.

\subsection{Self-reported Metrics}

From the data collected in interviews, it was possible to examine self-reported metrics quantitatively and qualitatively. Completion of the questionnaires using the Likert scale [27] is the basis of quantitative analysis presented below.

\subsection{1) Quantitative Analysis}

Fig. 14 shows the mean score for each device's usability, where 1 means "very bad" and 7 is "very good". The results of the analysis were consistent with results observed in the task duration metrics. The v-Glove without tactile feedback was the worst, followed by the glove with tactile feedback and the 3D mouse.

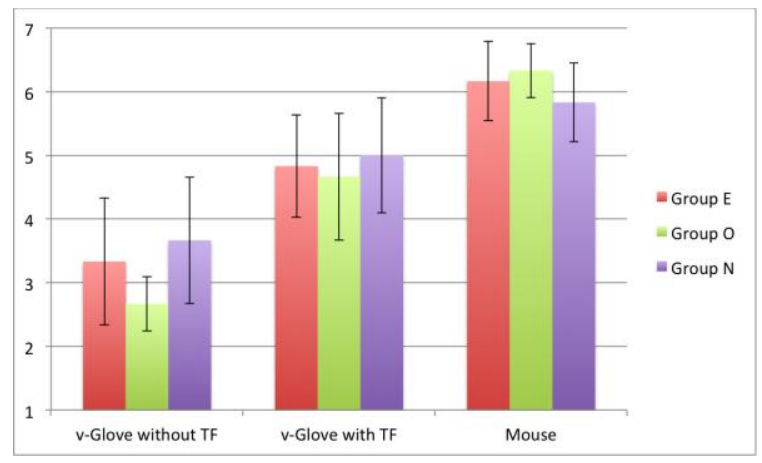

Fig. 14. Mean Points per Group.

\subsection{2) Qualitative Analysis}

Since most of the users had some experience in working with graphical software, during the interviews contributions from the participants were captured. The most common suggestions were:

- Increase the scale of the movement relative to the pointer on the screen;

- Use the click of the first contact with the touch area;

- $\quad$ Detect the click from a minimum variation in the $\mathrm{Z}$ axis after reaching the touch area;

- Decrease the intensity of vibration;

- Change the click way to reduce hand and arm fatigue;

- Click via a pinch gesture;

- Recognize click movement from a relative location, and not just when the user gets some region;

- Convert the slider widget to a vertical orientation;

- Provide some haptic response when the cursor reaches the area of a widget that supports interaction;

- $\quad$ Provide progressive tactile feedback;

- Include a click button in the v-Glove; 
- Keep vibration while the finger is in the click area.

Most feedback from the users was about tasks that require some precision, like adjusting the slider widget or selecting an object in a small area of the screen. These issues are partially related to characteristics of the proposed device, especially because the v-Glove was designed for free hand movements in space, where it is hard for a person to have movements with precision for a long time. However, improvements in the proposed widgets can be made to improve its usability. The slider widget could be redesigned in a vertical axis, increasing the corresponding interaction area for the $\mathrm{v}$-Glove.

\subsection{Discussion}

The task time metrics provided information with statistical relevance confirming that there are differences in task duration average for different devices. Despite a slight tendency towards a better performance according to the level of 3D experience of the users, the analysis of variance (ANOVA) of two factors with replication determined that this difference has no statistical significance when comparing the performance of one group over the other $(\mathrm{F}(3.20)=2.56, \mathrm{p}<0.09)$. The same technique also confirmed that the time differences between the devices are indeed significant $(\mathrm{F}(3.20)=19.19$, $\mathrm{p}<0.01)$, affirming the results obtained in earlier observations, when the mouse was expected to have the best performance followed by the v-Glove with tactile feedback.

Based on the results of the usability issues metrics it is also possible to foresee that some effort required to reduce the usability issues related to the v-Glove might result in improvements in the glove indicators compared to the mouse.

The tactile feedback had also a considerable impact in the v-Glove. The idea behind the inclusion of two versions of the $\mathrm{v}$-Glove in the tests was to study the influence of the tactile feedback in the glove usability. The results of the ANOVA (two factors with replication) applied to task time performance indicate a relevant statistical difference in favor of the v-Glove with tactile feedback $(\mathrm{F}(4.17)=5.23, \mathrm{p}<0.03)$ when compared to the v-Glove without feedback.

\section{CONCLUSIONS AND FUTURE WORK}

In this work, an interaction device for immersive virtual reality environments, called the v-Glove, was proposed and developed. The v-Glove is a device that allows a user to interact with virtual reality applications in a natural way through the movement of the hand in $3 \mathrm{D}$ space. Bringing the hand to a touch area mapped in the virtual space does the selection and manipulation of objects. As a proof of concept, an application capable of interacting with CAD data was adapted for an immersive environment. Some usability studies of the glove with quantitative and qualitative assessments were conducted with three groups of users with different profiles of 3D knowledge.

The use of gloves as an element of control pointer on the screen is more intuitive than the mouse itself, but because the interaction tasks supported by windowing applications have already been mapped to a 2D model to facilitate its use with the mouse, the graphical user interface has to be adapted. Applications designed for the standard WIMP can hardly be used in such an environment without some kind of adaptation.

Regarding the graphical user interface components studied, it was found that the slider widget is the hardest component to be adapted, regardless of the device used for interaction. In the combo box component, some interaction problems were also identified, since the user requires at least two clicks to select an option. Alternative formats such as those proposed by Gerber and Bechmann [28] and Dachselt and Ebert [29] might be considered. These authors suggest the adoption of, respectively, a ring menu and a cylindrical menu (called collapsible cylindrical trees).

During the evaluation of the tactile feedback, it was noted that this type of feedback is indeed essential to the v-Glove operation. This result was confirmed in comparative testing of the glove with and without this feature. Some improvements can still be made to this functionality, such as support to the varying intensity of the vibration and the progressive implementation of tactile feedback to better fit user expectations.

One of the most important features to be included is the support to multi-touch interaction and feedback. Recognition of gestures for the interaction tasks is another important feature to be considered. A more complete second glove is being produced to be used for left hand users and also allowing interaction with both hands simultaneously. This will improve the interactions, especially for tasks such as manipulating 3D objects in the scene. Once the 3D mouse used in our evaluation is a product very well designed by professionals, building the v-Glove over a commercial haptic glove as the one provided by [30] was also considered to improve the sense of quality, as well as the use of Kinect [31] instead of the WiiMote as tracking device.

Another resource planned for development is incorporating clicking via a pinch between the thumb and fingers, as found in the pinch gloves [32]. The glove would have to be modified including two electrical contacts at the thumb and index fingertips to close a circuit when in contact with each other.

The system is also being ported to a highly immersive system [33]. Due to the larger dimensions, new studies are being conducted in order to better find a position for the WiiMotes, not occluding the user view (Fig. 15).

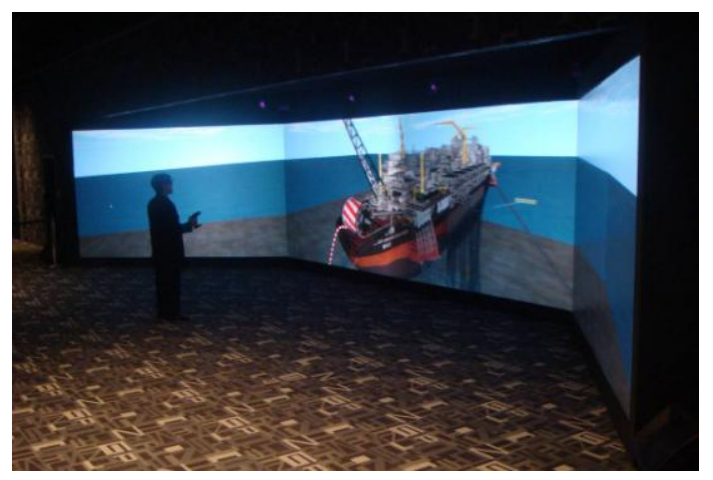

Fig. 15. High Immersive System Being Tested (Color Plate 3). 


\section{ACKNOWLEDGEMENTS}

Tecgraf is a laboratory mainly funded by Petrobras. Alberto Raposo receives a grant from FAPERJ E-26/102273/2009.

\section{REFERENCES}

[1] F. Bacim, D. Bowman, M. Pinho, Wayfinding Techniques for MultiScale Virtual Environments, IEEE Symposium on 3D User Interfaces, pp. 67-74, Louisiana, EUA, 2009.

[2] A. Kulik, J. Hochstrate, A. Kunert, B. Froelich, The Influence of Input Device Characteristics on Spatial Perception in Desktop-Based 3D Applications. IEEE Symposium on 3D User Interfaces, pp. 59-66, Louisiana, USA, 2009.

[3] S. Smith, S. Du'mont, Measuring the Effect of Gaming Experience on Virtual Environment Navigation Tasks. IEEE Symposium on 3D User Interfaces, pp. 3-10, Louisiana, EUA, 2009.

[4] D. Bowman, C. Wingrave, Design and Evaluation of Menu Systems for Immersive Virtual Environments, IEEE Virtual Reality Conference, pp 149-156, Yokohama, Japan, 2001.

[5] C. Hand, A Survey of 3D Interaction Techniques. Computer Graphics Forum, 16(5), pp. 269-281, December 1997.

[6] Jian Chen, Michael A. Narayan, Manuel A. Perez-Quinones, The Use of Hand-held Devices for Search Tasks in Virtual Environments, Proceedings of 3DUI, IEEE Virtual Reality, pp. 91-98, 2005.

[7] P. G. de Barros, R. J. Rolleston, R. W. Lindeman. Evaluation of Multi-Touch Techniques for Physically Simulated Virtual Object Manipulations in 3D Space, Proc. of the 6th IASTED Int'l Conf. on Human-Computer Interaction (HCI2011), 2011.

[8] R. Scheibe, M. Moehring, B. Froehlich, Tactile Feedback at the Finger Tips for Improved Direct Interaction in Immersive Environments. Virtual Reality Conference, pp. 123-130, Charlotte, NC, March 2007.

[9] C. Bullion, H. Gurocak, Haptic Glove with MR Brakes for Distributed Finger Force Feedback, Presence: Teleoperators and Virtual Environments, 18(6), pp. 421-433, February 2010.

[10] Huimin Qian, Ravi Kuber, and Andrew Sears, Towards developing perceivable tactile feedback for mobile devices, International Journal of Human-Computer Studies, 69(11), October 2011, pp. 705-719.

[11] ART Fingertracking, http://www.ar-tracking.de/Fingertracking. 54+B6Jkw9.0.html. Accessed January 2011

[12] T. Grossman, D. Wigdor, R. Balakrishnan, Multi-Finger Gestural Interaction with 3D Volumetric Displays, UIST, pp. 61-70, New Mexico, USA, 2004.

[13] M. Tosas, B. Li, Virtual Touch Screen for Mixed Reality. ECCV 2004 Workshop on HCI, pp. 48-59, Prague, Czech Republic, May 2004.

[14] R. W. Lindeman, J. L. Sibert, J. K. Hahn, Hand-Held Windows: Towards Effective 2D Interaction in Immersive Virtual Environments, Proceedings of IEEE Virtual Reality 1999, pp. 205-212.

[15] L. Dipietro, A. Sabatini, P. Dario, A Survey of Glove-Based Systems and Their Applications, IEEE Transactions on Systems, Man and Cybernetics, 38(4), July 2008.

[16] L. Buechley, M. Eisenberg, J. Catchen, A. Crockett, The LilyPad Arduino: Using Computational Textiles to Investigate Engagement, Aesthetics, and Diversity in Computer Science Education, CHI 2008, pp. 423-432, Florence, Italy.

[17] Xbee,http://www.digi.com/products/wireless/point-multipoint/xbee-serie s1-module.jsp\#docs. Accessed January 2011.

[18] Tune Glove Diagram, http://www.flickr.com/photos/jtanenbaum/ 4156417140/.Accessed January 2011.

[19] Johnny Chung Lee. 2008. Hacking the Nintendo Wii Remote. IEEE Pervasive Computing 7, 3 (July 2008), pp. 39-45. DOI=10.1109/MPRV. 2008.53.

[20] Lowe, David G., Distinctive Image Features from Scale-Invariant Keypoints, International Journal of Computer Vision, pp. 91-110, Springer Netherlands.
[21] P. C. Santos, A. Stork, A. Buaes, C. E. Pereira, Jorge: A real-time low-cost marker-based multiple camera tracking solution for virtual reality applications. J. Real-Time Image Processing 5(2): pp. 121-128, 2010.

[22] A. Raposo, I. Santos, L. Soares, G. Wagner, E. Corseuil, M. Gattass, Environ: Integrating VR and CAD in Engineering Projects, IEEE Computer Graphics \& Applications, 29(6), pp. 91-95, 2009.

[23] J. Nielsen, Why you only need to test with 5 users. Alertbox, 2000, www.useit.com/alertbox/2000319.html.

[24] R. Virzi, Refining the test phase of usability evaluation: How many subjects is enough? Human Factors 34, pp. 457-468, 1992.

[25] J.R. Lewis, Sample sizes for usability studies: Additional considerations. Human Factors 36, pp. 368-378, 1994.

[26] Air Mouse Go Plus, http://www.gyration.com/index.php/us/products/ in-air-micekeyboards/go-air-mouse.html. Accessed January 2011.

[27] T. Tullis, B. Albert 2008, Measuring the User Experience, Morgan Kaufmann (ISBN-13 978-0-12-373558-4), USA, 2008.

[28] D. Gerber, D. Bechmann, Design and evaluation of the ring menu in virtual environments. IPT 2004: Eighth immersive projection technology workshop, Ames, IA, USA, 2004.

[29] R. Dachselt, J. Ebert, Collapsible cylindrical trees: a fast hierarchical navigation technique. Proceedings of the IEEE Symposium on Information Visualization 2001 (INFOVIS'01). Washington, DC, EUA, pp. $79-86$.

[30] CyberGlove, http://www.vrealities.com/cybertouch.html. Accessed April 2011.

[31] Kinect, http://www.xbox.com/en-US/kinect. Accessed September 2011.

[32] D. A. Bowman, C. A. Wingrave, J. M. Campbell, and V. Q. Ly, Using Pinch Gloves for both Natural and Abstract Interaction Techniques in Virtual Environments, Proceedings of HCI 2001, pp. 629-633.

[33] C. Cruz-Neira, D. Sandin, T. Defanti, R. Kenyon, J. Hart, "The CAVE: Audio Visual Experience Automatic Virtual Environment," Communications of the ACM, 35(6), pp. 64-72, June 1992.

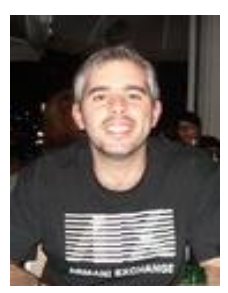

Paulo Gallotti Rodrigues holds an MSc in Information Systems from Pontifícia Universidade Católica do Rio de Janeiro. His research interests include 3D interaction in immersive environments, ubiquitous computing and collaboration systems. He is currently a researcher at the Tecgraf, Computer Graphics Technology Group in PUC-Rio working in several projects with Petrobras.

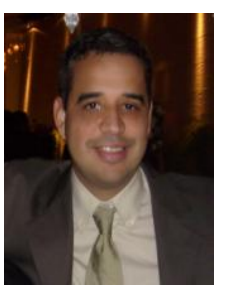

Alberto Barbosa Raposo is Assistant Professor at the Dept. of Informatics / PUC-Rio, project coordinator at Tecgraf/PUC-Rio and FAPERJ researcher. DSc in Electrical/Computer Engineering at the State University of Campinas, Brazil. Current interests: Virtual Reality, 3D interaction, groupware, $\mathrm{HCI}$, and computer graphics, with more than 120 publications in these areas. Projects supported by: Petrobras, CNPq, FINEP, FAPERJ and RNP. Distinguished young scholar, PUC-Rio and NVIDIA

Academic Partner.

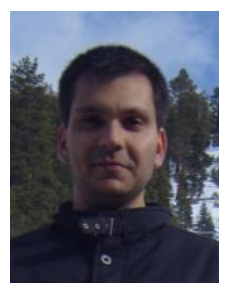

Luciano Pereira Soares holds a PhD in Electrical Engineering from Polytechnic School, University of São Paulo in Brazil. He was a postdoctoral research at the Instituto Superior Técnico in Portugal, Institut National de Recherche en Informatique et en Automatique in France and Instituto Superior de Ciências do Trabalho e da Empresa in Portugal. His research interests include, immersive multi-projection systems, real-time 3D computer graphics and high performance computing. He is currently a researcher at the Tecgraf, Computer Graphics Technology Group in PUC-Rio working in several projects with Petrobras, and Associate Professor at the Centro Universitário da Cidade do Rio de Janeiro. 